Boletín de la Sociedad Zoológica del Uruguay, 2020

Vol. 29 (2): 86-98

ISSN 2393-6940

https://journal.szu.org.uy

\title{
HISTERIDAE (COLEOPTERA) DE URUGUAY: LISTA PRELIMINAR DE ESPECIES, NUEVOS REGISTROS Y DISTRIBUCIÓN POTENCIAL
}

\author{
Ana Belén Aguilar-Sosa, Gabriel Morales-Díaz, Leonel Basualdo, Romina Roibal y Patricia González- \\ Vainer
}

Sección Entomología, Facultad de Ciencias, Universidad de la República. Mataojo 4225, 11400 Montevideo, Uruguay.

Autor para correspondencia: vainer@fcien.edu.uy

\begin{abstract}
RESUMEN
Los Histéridos son escarabajos predadores asociados principalmente a cadáveres y estiércol, ya que se alimentan de huevos y larvas de otros insectos. Son de interés en la investigación forense y como controladores de especies parásitas, especialmente moscas. Se presenta un inventario actualizado de las especies de la familia en Uruguay, así como un análisis de distribución en el territorio. Treinta y siete especies y 13 morfoespecies de 13 géneros se incluyeron en el inventario con información de hábitat y microhábitat. Se registraron 5 nuevas especies por primera vez para Uruguay: Hololepta humilis, Carcinops troglodytes, Hister lissurus, Hister cavifrons y Phelister pumilus. Se ingresaron a la Colección de Entomología de la Facultad de Ciencias representantes de todas las especies, al menos un individuo por cada sitio de colecta. Las especies más abundantes fueron Euspilotus azureus, Euspilotus connectens y Phelister pumilus. La familia tiene una amplia distribución en el territorio nacional, con valores de favorabilidad mayores en el litoral sur.
\end{abstract}

Palabras clave: Euspilotus, Hister, Hololepta, Phelister

\begin{abstract}
Histeridae (Coleoptera) from Uruguay: updated list of species, new records and potential distribution. Histerids are predatory beetles mainly associated with corpses and faeces as they feed on other insects' eggs and larvae. They are of interest in forensic investigation and as controllers of parasite species, mostly flies. An actualized inventory of the species of the family in Uruguay is presented, as well as an analysis of the distribution in the territory. Thirty-seven species and 13 morphospecies of 13 genus were included in the inventory with habitat and microhabitat information. Five species are recorded for the first time for Uruguay: Hololepta humilis, Carcinops troglodytes, Hister lissurus, Hister cavifrons and Phelister pumilus. Representatives of all species, at least one individual for each collection site, were deposited into the
\end{abstract}

Entomology Collection of the Faculty of Sciences. The most abundant species were Euspilotus azureus, Euspilotus connectens and Phelister pumilus. The family has a wide distribution in the national territory, with higher favorability values on the southern coast.

Key words: Euspilotus, Hister, Hololepta, Phelister

\section{INTRODUCCIÓN}

Histeridae Gyllenhaal, 1808, es una familia de distribución mundial. Comprende 4252 especies y 391 géneros, agrupados en 11 subfamilias (Mazur, 2011); en la región Neotropical se han registrado 139 géneros y 1047 especies (Almeida and Mise, 2009). Se caracterizan por ser insectos pequeños, de cuerpo compacto y coloración oscura, con élitros cortos dejando expuestos los últimos dos tergitos abdominales (raramente uno) y la cabeza generalmente retraída debajo del protórax. Son insectos predadores tanto en estado adulto como larval y están asociadas a diversos medios: cadáveres en descomposición, excrementos, restos vegetales y debajo de corteza de árboles muertos (Bentancourt et al., 2009). También pueden vivir en simbiosis en nidos de vertebrados y en colonias de insectos sociales (Fernández et al., 2005). Se alimentan mayormente de huevos y larvas de cuerpo blando de otros insectos, especialmente de dípteros Muscomorpha, cuyas larvas se desarrollan en cadáveres y excrementos de grandes mamíferos, por lo cual son de especial interés en la investigación forense y como controladores de especies de moscas (Aballay et al., 2013). Las especies de histéridos atraídas a los cadáveres, completan todo su ciclo de vida en el cuerpo en descomposición, constituyendo buenos indicadores para la determinación del Intervalo Post Mortem (IPM), principal objetivo de la Entomología Forense (Aballay et al., 2013). Los imagos alcanzan su máxima abundancia en cuerpos en estados avanzados de 
descomposición, por lo que son especialmente útiles para establecer el IPM cuando el cadáver es hallado mucho tiempo después de ocurrida la muerte (Özdemir \& Sert, 2009).

Algunas especies de histéridos son enemigos naturales de determinadas especies de moscas como Musca domestica Linnaeus (Muscidae) y Chrysomya putoria (Wiedemann) (Calliphoridae) (Aballay et al., 2013), ambas transmisoras de diversos agentes patógenos para el ser humano y la segunda es también causante de miasis en el hombre y animales domésticos (Greenberg \& Szyska, 1984). Varias especies han sido identificadas también como controladores de moscas plaga que se desarrollan en los excrementos vacunos (Cicchino, 1999). Otros histéridos son considerados agentes potenciales de control biológico para el coleóptero Alphitobius diaperinus Panzer (Tenebrionidae) que se desarrolla en las heces de pollo y constituye una de las principales plagas a nivel mundial en las explotaciones avícolas (Santoro et al. 2010). Por otra parte, algunas especies de histéridos viven dentro de colmenas de abejas meliponas (Hymenoptera: Apidae), predando sobre sus larvas y pupas, representado un problema en la producción apícola (Coletto-Silva \& Freire, 2006).

En Uruguay no se ha realizado ningún tipo de estudio enfocado en la diversidad, biología, taxonomía o distribución geográfica de las especies de Histeridae. El conocimiento sobre las especies de esta familia presentes en el país es escaso y fragmentario. Monné (1970) realiza un listado de las especies de coleópteros presentes en Uruguay, citando 27 especies de Histeridae de 11 géneros y 5 subfamilias, de las cuales sólo seis especies estaban representadas en la Colección de Entomología (Coleoptera) de la Facultad de Ciencias (FCE-Co). En Argentina se han registrado 57 especies de 39 géneros (Arriagada, 2013) mientras que en la región Sur de Brasil se han citado 157 especies de 66 géneros (Gonçalves \& Leivas, 2017). Considerando la riqueza de especies existente a nivel regional, es de esperar que la riqueza de Histeridae en Uruguay sea mayor a la ya conocida. Desde el punto de vista ecológico, un estudio sobre coleópteros sarcosaprófagos realizado en un paisaje de serranía de Uruguay demostró que hay especies de histéridos típicas de bosques nativos y otras que prefieren el pastizal (González-Vainer \& Morelli 2008; GonzálezVainer et al., 2012). De allí se desprende la necesidad de realizar relevamientos de estos insectos en diferentes ambientes para ampliar el conocimiento sobre su riqueza.

El objetivo del presente estudio es realizar un inventario actualizado de las especies de Histeridae de Uruguay, aportando para cada una los datos georeferenciados de su distribución en el país y sobre los hábitats y microhábitats asociados. Se realiza además un análisis preliminar de la distribución geográfica potencial de la familia y las especies más abundantes en el país.

\section{MATERIAL Y MÉTODOS}

Se realizó una revisión bibliográfica exhaustiva de publicaciones posteriores al catálogo de Monné (1970) para detectar especies citadas para Uruguay.

Se realizaron 6 salidas de campo cubriendo 17 de los 19 Departamentos de Uruguay durante los meses de otoño, primavera y verano de 2019. Se recolectaron individuos mediante colecta manual directa en heces de bovino y equino, cadáveres y bajo corteza. También se colocaron trampas de caída cebadas con carroña. Fueron tomadas coordenadas por medio de GPS en cada punto de colecta.

Se revisaron los ejemplares de Histeridae depositados en la Colección de Entomología (Coleoptera) de la Facultad de Ciencias (FCE-Co) y aquellos de muestras disponibles en la Sección de Entomología de la Facultad de Ciencias, provenientes de diversos estudios anteriores, la mayoría no identificados previamente. La identificación a nivel de especies se realizó utilizando las claves para géneros y especies de Mazur (2001), Aballay et al. (2013) y Caterino \& Tishechkin (2019); también se revisaron descripciones originales de las especies (Marseul, 1853, 1854, 1870; Lewis, 1897; Bickhardt, 1909; Arriagada, 2018) para aquellos casos en que no había claves disponibles.

Ejemplares de todas las especies, uno de cada sitio de colecta, fueron depositados en seco (pinchados) en la Colección de Entomología de Facultad de Ciencias. El resto de los especímenes fueron depositados en seco (en sobres) y en alcohol $70 \%$.

Las especies que representan nuevos registros para Uruguay fueron fotografiadas y se realizó una breve descripción de las mismas, particularmente de aquellos caracteres que permitieron identificarlas. Se utilizaron como base las descripciones originales y se tuvieron en consideración las sinonimias.

Se elaboró una base de datos georreferenciada de las especies presentes en Uruguay, con la correspondiente información de colecta en Libre Office v6.3.0.4 (The Document Foundation 2019) (Apéndice 1). Para cada uno de los registros inventariados sin información georreferenciada, se tomaron las coordenadas de la localidad, zona o punto de referencia a partir de la información disponible. Fueron utilizados los siguientes geoservicios disponibles para mayor precisión, MTOP Open Data (versión 1.2.1 - Equipo IDE MTOP) para referencias viales, Sistema de información territorial (MVOTMA - Dirección Nacional de ordenamiento Territorial) para localidades e información disponible en la web para puntos de referencia. 
Se estimaron áreas favorables para la familia y las tres especies con más ocurrencias, por medio de modelos de distribución. La superficie del territorio de Uruguay se dividió en una malla de cuadrados de $10 \times 10 \mathrm{~km}$, utilizando herramientas de QGIS v3.8.1 (QGIS Development Team, 2019), obteniendo 1883 unidades. Fueron utilizadas todas las presencias disponibles y los promedios de 19 variables predictoras obtenidas de WorldClim 2 (Fick \& Hijmans, 2017, ver Tabla 1). Como algoritmo de modelaje, se utilizó la función de favorabilidad (Real et al., 2006), por medio de la librería fuzzySim v3.0 (Barbosa, 2015) implementada en el programa $R(R$ Core Team 2019). La función multGLM fue usada para construir secuencial y conjuntamente los 4 modelos. Para seleccionar las variables, a través de la función FDR, se controló el incremento en el error de tipo I en la hipótesis nula, usando la tasa de falso descubrimiento (FDR) por el procedimiento de Benjamini \& Hochberg (1995). Posteriormente, tanto las funciones step (Hastie \& Pregibon, 1992), que realizan una selección de variables paso a paso y aplican el criterio de información de Akaike (AIC, Akaike, 1998), así como la función modelTrim (Barbosa, 2015), que realiza una selección paso a paso que elimina variables no significativas, fueron utilizadas para finalizar el filtrado de variables para el modelo. Los modelos fueron evaluados simultáneamente por medio de la librería modEvA v2.0 (Barbosa et al., 2014). Partiendo de un valor de favorabilidad de $F=0.5$ como umbral de clasificación (Real et al., 2006), se evaluó la capacidad de clasificación de los modelos, mediante la sensibilidad y tasa de clasificación correcta (CCR, Fielding \&
Bell, 1997). También se calculó la capacidad de discriminación mediante el área bajo la curva (AUC) (Fielding \& Bell, 1997), sin definirse ningún umbral (Hosmer \& Lemeshow, 2000).

\section{RESULTADOS}

A partir de 1139 ejemplares de Histeridae examinados, y de la recopilación bibliográfica, se inventariaron 37 especies y 13 morfoespecies identificadas a nivel de género, distribuídas en 13 géneros y 5 subfamilias. Se registraron cinco nuevas especies para Uruguay: Hololepta humilis Paykull, 1811; Carcinops troglodytes Paykull, 1811; Hister lissurus Marseul, 1854; Hister cavifrons Marseul, 1854 y Phelister pumilus Erichson, 1834.

En la Tabla 2 se incluye la lista actualizada de especies con la información de hábitat y microhábitat conocida. Las especies más abundantes fueron Euspilotus azureus (Sahlberg), Euspilotus connectens (Paykull) y Phelister pumilus Erichson con 497, 223 y 212 individuos, representando las tres el $82 \%$ del total de individuos. Estas especies se encontraron en más de la mitad de los departamentos del país, tanto en ambientes abiertos de pastizal como en bosques nativos y exóticos. $P$. pumilus se encontró exclusivamente en carroña; $E$. azureus y $P$. connectens se encontraron tanto en estiércol como carroña, pero E. azureus presentó una marcada preferencia por carroña mientras que $P$. connectens fue más abundante en estiércol.

El pastizal fue el ambiente con más especies registradas, seguido de las plantaciones de eucaliptus.

Tabla 1. Lista de variables bioclimáticas utilizadas para elaborar los modelos de favorabilidad de la familia y las tres especies con más registros (T: temperatura).

\section{Variables}

T media anual $\left({ }^{\circ} \mathrm{C}\right)$

Variación de T diurna $\left({ }^{\circ} \mathrm{C}\right)$

Isotermalidad (\%)

Estacionalidad de la T (\%)

T máxima del mes más cálido $\left({ }^{\circ} \mathrm{C}\right)$

T mínima del mes más frío $\left({ }^{\circ} \mathrm{C}\right)$

Rango anual de $\mathrm{T}\left({ }^{\circ} \mathrm{C}\right)$

$\mathrm{T}$ media del trimestre más húmedo $\left({ }^{\circ} \mathrm{C}\right)$

$\mathrm{T}$ media del trimestre más seco $\left({ }^{\circ} \mathrm{C}\right)$

T media del trimestre más cálido $\left({ }^{\circ} \mathrm{C}\right)$

\section{Código Variables}

Código

$\begin{array}{lll}\mathrm{BIO} 1 & \text { T media del trimestre más frío }\left({ }^{\circ} \mathrm{C}\right) & \mathrm{BIO} 11 \\ \mathrm{BIO} 2 & \text { Precipitación anual }(\mathrm{mm}) & \mathrm{BIO} 12 \\ \mathrm{BIO} 3 & \text { Precipitación del mes más húmedo }(\mathrm{mm}) & \mathrm{BIO} 13 \\ \mathrm{BIO} 4 & \text { Precipitación del mes más seco }(\mathrm{mm}) & \mathrm{BIO} 14 \\ \mathrm{BIO} 5 & \text { Estacionalidad de la precipitación }(\%) & \mathrm{BIO}) \\ \mathrm{BIO} 6 & \text { Precipitación del trimestre más húmedo }\left({ }^{\circ} \mathrm{C}\right) & \mathrm{BIO16} \\ \mathrm{BIO} 7 & \text { Precipitación del trimestre más seco }\left({ }^{\circ} \mathrm{C}\right) & \mathrm{BIO17} \\ \mathrm{BIO}) & \text { Precipitación del trimestre más cálido }\left({ }^{\circ} \mathrm{C}\right) & \mathrm{BIO18} \\ \mathrm{BIO}) & \text { Precipitación del trimestre más frío }\left({ }^{\circ} \mathrm{C}\right) & \mathrm{BIO19}\end{array}$

$\mathrm{BIO10}$ 
Tabla 2. Listado de especies y morfoespecies con el detalle de hábitat y microhábitat. (BC: bajo corteza; BI: blanqueal; BR: bosque ribereño; BS: bosque serrano; C: cadáver; Es: estiércol; Eu: eucaliptal; H: hormiguero; P: pastizal; Pi: pinar; Pm: palmar; VD: vegetales en descomposición)

\begin{tabular}{|c|c|c|c|c|c|c|c|c|c|c|c|c|c|}
\hline \multirow[b]{2}{*}{$\begin{array}{l}\text { Subfamilia } \\
\text { Especie }\end{array}$} & \multirow[b]{2}{*}{$\begin{array}{c}\text { Distribución } \\
\text { (Departamentos) }\end{array}$} & \multirow[b]{2}{*}{ Fuente } & \multirow[b]{2}{*}{ BS } & \multirow[b]{2}{*}{ BR } & \multicolumn{3}{|c|}{ Hábitats } & \multirow[b]{2}{*}{ BI Pm } & \multirow[b]{2}{*}{ BC } & \multicolumn{3}{|c|}{ Microhábitats } & \multirow[b]{2}{*}{ Es } \\
\hline & & & & & $\mathbf{P}$ & Eu & $\mathbf{P i}$ & & & VD & H & C & \\
\hline \multicolumn{14}{|l|}{ Hololeptinae } \\
\hline $\begin{array}{l}\text { Hololepta aradiformis } \\
\text { Erichson }\end{array}$ & ma, mo & $\begin{array}{l}\text { Monné 1970; } \\
\text { este estudio }\end{array}$ & & $x$ & & & & & & $x$ & & & \\
\hline $\begin{array}{l}\text { Hololepta attenuata } \\
\text { Blanchard }\end{array}$ & & Monné 1970 & & & & & & & & & & & \\
\hline Hololepta humilis Paykul & mo & Este estudio & & & & & & & & & & & \\
\hline \multicolumn{14}{|l|}{ Saprininae } \\
\hline $\begin{array}{l}\text { Euspilotus azurescens } \\
\text { Marseul }\end{array}$ & $\mathrm{ca}, \mathrm{mo}$ & $\begin{array}{l}\text { Blackwelder 1944; } \\
\text { este estudio }\end{array}$ & & & & & & & & & & & \\
\hline $\begin{array}{l}\text { Euspilotus azureus } \\
\text { Sahlberg }\end{array}$ & $\begin{array}{l}\text { ar, ro, rv, du, rn, } \\
\text { ca, la, pa, ta, fd }\end{array}$ & $\begin{array}{l}\text { Castro et al. 2019; } \\
\text { Este estudio }\end{array}$ & $x$ & $x$ & $x$ & $X$ & & $x$ & & & & $x$ & $x$ \\
\hline $\begin{array}{l}\text { Euspilotus connectens } \\
\text { Paykull }\end{array}$ & $\begin{array}{l}\text { mo, ro, la, du, fd, } \\
\text { ca, fs, pa, rn, } \\
\text { sj, so, cl }\end{array}$ & $\begin{array}{l}\text { Monné 1970; } \\
\text { Remedios 2014; } \\
\text { Castro et al. 2019; } \\
\text { este estudio }\end{array}$ & $x$ & & $x$ & $x$ & & $x$ & & & & $x$ & $x$ \\
\hline \multicolumn{2}{|l|}{ Euspilotus eremita Marseul } & Monné 1970 & & & & & & & & & & & \\
\hline \multicolumn{2}{|l|}{ Euspilotus limatus (Marseul) } & Blackwelder 1944 & & & & & & & & & & & \\
\hline $\begin{array}{l}\text { Euspilotus modestus } \\
\text { (Erichson) }\end{array}$ & $\begin{array}{l}\text { ca, pa, ro, rn, sj, } \\
\text { co, du }\end{array}$ & $\begin{array}{l}\text { Monné 1970; } \\
\text { Remedios 2014; } \\
\text { Castro et al. 2019; } \\
\text { Este estudio }\end{array}$ & $x$ & & $x$ & & & & & & & $x$ & $x$ \\
\hline \multicolumn{2}{|l|}{$\begin{array}{l}\text { Euspilotus myrmercophilus } \\
\text { Bickhardt }\end{array}$} & Blackwelder 1944 & & & & & & & & & & & \\
\hline $\begin{array}{l}\text { Euspilotus pavidus } \\
\text { (Erichson) }\end{array}$ & $\mathrm{rv}, \mathrm{ro}, \mathrm{mo}, \mathrm{ma}, \mathrm{fd}$ & $\begin{array}{l}\text { Monné 1970; } \\
\text { Este estudio }\end{array}$ & & & $x$ & & & & & & & $x$ & $x$ \\
\hline Euspilotus rubriculus Marseul & mo & Monné 1970 & & & & & & & & & & & \\
\hline Euspilotus strobeli (Steinheil) & $\mathrm{ca}$ & Este estudio & & & & $X$ & & & & & & $X$ & \\
\hline Euspilotus sp1 & $\mathrm{fd}$ & Este estudio & & & $x$ & $X$ & & & & & & $X$ & $X$ \\
\hline Euspilotus sp2 & $\mathrm{fd}$ & Este estudio & & & $x$ & & & & & & & & $X$ \\
\hline Euspilotus sp3 & $\mathrm{fd}$ & Este estudio & & & $x$ & & & & & & & & $x$ \\
\hline Euspilotus sp4 & ma & Este estudio & & & & & & & & & & & \\
\hline Euspilotus sp5 & la & Este estudio & & $X$ & & & & & & & & $X$ & \\
\hline Euspilotus sp6 & la & Este estudio & & & & & $x$ & & & & & & $x$ \\
\hline $\begin{array}{l}\text { Hypocaccus brasiliensis } \\
\text { Paykull }\end{array}$ & sj, ma & $\begin{array}{l}\text { Monné 1970; } \\
\text { Este estudio }\end{array}$ & & & & & & & & & & $x$ & \\
\hline Tatianella dolata (Marseul) & ma & Arriagada, 2018 & & & & & & & & & & $x$ & \\
\hline \multicolumn{14}{|l|}{ Dendrophilinae } \\
\hline Carcinops troglodytes Paykull & $\mathrm{ca}, \mathrm{mo}$ & $\begin{array}{l}\text { Remedios, 2014; } \\
\text { Este estudio }\end{array}$ & $x$ & & & $x$ & & & $x$ & & & $x$ & $x$ \\
\hline Carcinops sp1 & mo, rv & Este estudio & $x$ & & & & & & $x$ & $x$ & & & \\
\hline Paromalus oculipygus Marseul & & Blackwelder 1944 & & & & & & & & & & & \\
\hline Paromalus sp1 & mo & Este estudio & & & & & & & & & & & $x$ \\
\hline Histerinae & & & & & & & & & & & & & \\
\hline $\begin{array}{l}\text { Hister cavifrons } \\
\text { Marseul }\end{array}$ & $\begin{array}{l}\text { co, du, fd, fs, la, } \\
\text { pa, rn, rv, sj }\end{array}$ & Este estudio & $x$ & $x$ & $x$ & $x$ & $x$ & & & & & $x$ & $x$ \\
\hline
\end{tabular}


Tabla 2 (Continuación)

\begin{tabular}{|c|c|c|c|c|c|c|c|c|c|c|c|c|c|}
\hline \multirow[b]{2}{*}{$\begin{array}{l}\text { Subfamilia } \\
\text { Especie }\end{array}$} & \multirow[b]{2}{*}{$\begin{array}{c}\text { Distribución } \\
\text { (Departamentos) }\end{array}$} & \multirow[b]{2}{*}{ Fuente } & \multirow[b]{2}{*}{ BS } & \multirow[b]{2}{*}{ BR } & \multicolumn{3}{|c|}{ Hábitats } & \multirow[b]{2}{*}{ BI Pm } & \multirow[b]{2}{*}{ BC } & \multicolumn{3}{|c|}{ Microhábitats } & \multirow[b]{2}{*}{ Es } \\
\hline & & & & & $\mathbf{P}$ & Eu & $\mathrm{Pi}$ & & & VD & $\mathbf{H}$ & C & \\
\hline Hister dubius Marseul & $\mathrm{cl}, \mathrm{mo}, \mathrm{rn}$ & Blackwelder 1944 & & & & & & & & & & & \\
\hline Hister lissurus Marseul & $\mathrm{du}, \mathrm{fd}, \mathrm{so}, \mathrm{pa}$ & Este estudio & & & $X$ & $X$ & & & & & & $x$ & $X$ \\
\hline Hister platanus Marseul & mo, ca, ma & Monné, 1970 & & & & & & & & & & & \\
\hline Hister punctifer Paykull & Uruguay & Monné, 1970 & & & & & & & & & & & \\
\hline Hister sp 1 & $\mathrm{fd}$ & Este estudio & & & $x$ & & & & & & & & $x$ \\
\hline Hister sp2 & du & Este estudio & & & $x$ & $x$ & & & & & & & $x$ \\
\hline Kaszabister ferrugineus Kirsc & sch & Mazur 2011 & & & & & & & & & $x$ & & \\
\hline Kaszabister rubellus Erichson & rv & Mazur 2011 & & & & & & & & & $X$ & & \\
\hline Omalodes ebeninus Erichson & mo & Erichson, 1834 & & & & & & & & & & & \\
\hline Omalodes Iucidus Erichson & Uruguay & Mazur 2011 & & & & & & & & & & & \\
\hline Omalodes marseuli Schmidt & Uruguay & Mazur 2011 & & & & & & & & & & & \\
\hline Omalodes sp1 & mo & Este estudio & & & & & & & & & & & \\
\hline Phelister fulvulus Marseul & du & $\begin{array}{l}\text { Monné 1970; } \\
\text { Este estudio }\end{array}$ & & & $x$ & & & & & & & & $x$ \\
\hline $\begin{array}{l}\text { Phelister haemorrhous } \\
\text { Marseul }\end{array}$ & mo, sj & $\begin{array}{l}\text { Monné 1970; } \\
\text { Este estudio }\end{array}$ & & & $X$ & & & & & & & & $X$ \\
\hline $\begin{array}{l}\text { Phelister muscícapa } \\
\text { Marseul }\end{array}$ & $\begin{array}{c}\text { art, ca, du, mo, ri } \\
\text { rn, art }\end{array}$ & $\begin{array}{r}\text { ri, } \quad \text { Monné 1970, } \\
\text { Este estudio }\end{array}$ & & & $x$ & & & & & & $x$ & & $x$ \\
\hline $\begin{array}{l}\text { Phelister pumilus } \\
\text { Erichson }\end{array}$ & $\begin{array}{l}\text { fd, ca, rn, ta, la, } \\
\text { so, sj, fs, ro, du, r }\end{array}$ & mo Este estudio & & & $x$ & $x$ & & $x$ & & & & & $x$ \\
\hline $\begin{array}{l}\text { Phelister rufinotus } \\
\text { Marseul }\end{array}$ & rv & $\begin{array}{l}\text { Remedios 2014; } \\
\text { Este estudio }\end{array}$ & & & $x$ & & & & & & & & $x$ \\
\hline $\begin{array}{l}\text { Phelister stercoricola } \\
\text { Bickhardt }\end{array}$ & $\mathrm{ca}, \mathrm{mo}, \mathrm{rn}$ & $\begin{array}{l}\text { Blackwelder 1944; } \\
\quad \text { Monne } 1970\end{array}$ & & & & & & & & & & & \\
\hline $\begin{array}{l}\text { Phelister tremolerasi } \\
\text { Bickhardt }\end{array}$ & $\mathrm{rn}$ & Monné 1970 & & & & & & & & & & & \\
\hline Phelister sp1 & mo, ca & Este estudio & & & $x$ & $x$ & & & & & & $x$ & $x$ \\
\hline Phelister sp2 & $\mathrm{ca}$ & Este estudio & & & $x$ & $x$ & & & & & & $x$ & $x$ \\
\hline $\begin{array}{l}\text { Phelister sp3 } \\
\text { (Grupo haemorrhous) }\end{array}$ & $\mathrm{rn}$ & Este estudio & & & $x$ & & & & & & & & $x$ \\
\hline \multicolumn{14}{|l|}{ Hetariinae } \\
\hline $\begin{array}{l}\text { Chelonosternus tremolerasi } \\
\text { Bickhardt }\end{array}$ & mo & $\begin{array}{l}\text { Blackwelder 1944; } \\
\quad \text { Monne } 1970\end{array}$ & & & & & & & & & & & \\
\hline Termitolister koehleri Bruch & Uruguay & Mazur 2011 & & & & & & & & & & & \\
\hline $\begin{array}{l}\text { Reninus arechavaletae } \\
\text { Marseul }\end{array}$ & mo & $\begin{array}{l}\text { Blackwelder 1944; } \\
\text { Monne } 1970\end{array}$ & & & & & & & & & & & \\
\hline
\end{tabular}

Abreviaturas. ar: Artigas; ca: Canelones; cl: Cerro Largo; co: Colonia; du: Durazno; fs: Flores; fd: Florida; la: Lavalleja; ma: Maldonado; mo: Montevideo; pa: Paysandú; rn: Río Negro; rv: Rivera; ro: Rocha; sa: Salto; sj: San José; so: Soriano; ta: Tacuarembó. 


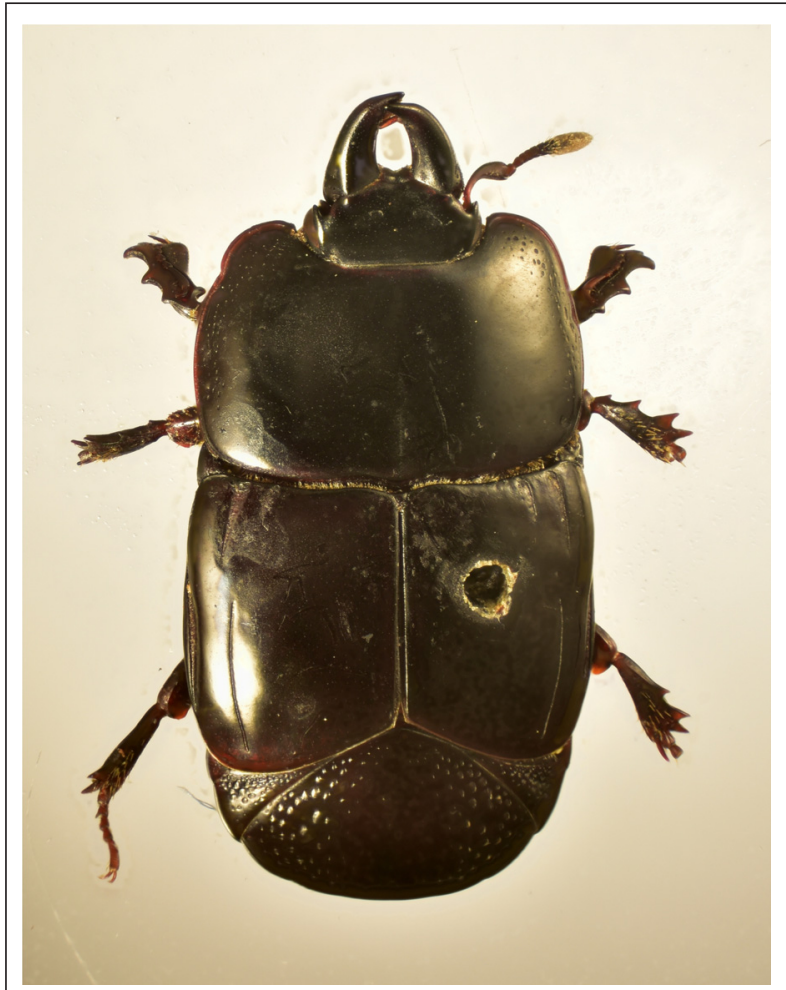

Fig. 1. Hololepta humilis en vista dorsal. Foto por D. Hagopián.

\section{Breve descripción de las especies citadas por primera vez para Uruguay}

\section{Hololepta humilis Paykull, 1811 (Fig. 1)}

Cuerpo oblongo y ligeramente aplanado (largo: $8,5 \mathrm{~mm}$, ancho: $4,5 \mathrm{~mm}$ ). Color negro brillante, antenas marrones y maza gris. Frente finamente punteada, con dos crestas transversales arqueadas, diente preocular prominente. Pronoto finamente punteado, con puntuación más gruesa a los lados; margen bisinuoso en la base, curvo y liso a los lados; estría longitudinal media acortada a la mitad; estría marginal marcada y en ángulo. Élitros del mismo ancho que la base del pronoto, recto a los lados y oblicuo al final, con un ángulo sutural bien marcado; estría subhumeral gruesa y recortada en la base; dos estrías dorsales: la 1 reducida a la mitad anterior, la 2 interrumpida, representada por un corto fragmento basal que luego se continúa en la mitad posterior casi hasta el margen apical. Propigidio con puntos espaciados. Pigidio densamente punteado. Protibias con cuatro dientes, meso y metatibias con tres.

Distribución: Brasil, Argentina (Blackwelder, 1945); Ecuador, América Central (Mazur, 2011); Uruguay.

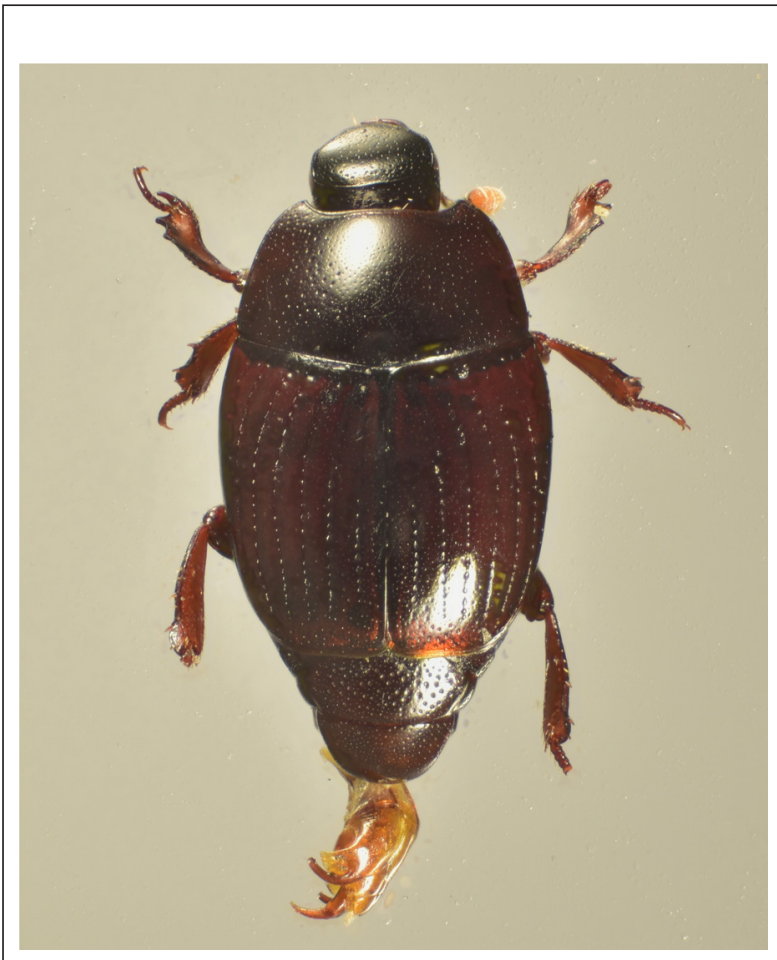

Fig. 2. Carcinops troglodytes en vista dorsal. Foto por B. Aguilar y D. Hagopián.

\section{Carcinops troglodytes (Paykull, 1811) (Fig. 2)}

Tamaño pequeño (largo: 2,1-2,3 mm; ancho: 1,3$1,6 \mathrm{~mm})$. Color negro brillante, antenas castañas con masa anaranjada, élitros y patas pardo-rojizas. Cuerpo ovalado, alargado. Pronoto con puntuación fina y escasa, puntos más anchos en la zona lateral, y una foseta en la parte media próximo al margen posterior. Élitros con puntuación fina y escasa en intervalos; estrías elitrales dorsales 1-5 completas, delimitadas con puntos bien marcados; estría sutural presente, reducida en la parte basal. Pigidio sin surcos. Protibia expandida, con 2 dentículos cortos separados por una concavidad y un espolón apical largo; el margen externo aserrado en la mitad proximal.

Distribución. Cosmopolita (Mazur, 2011); Argentina (Aballay et al., 2013); Uruguay.

\section{Hister lissurus Marseul, 1854 (Fig. 3)}

Cuerpo ovalado, ligeramente convexo (largo: 6,5 $\mathrm{mm}$; ancho 5,0 mm). Color negro brillante, antenas marrones con maza gris-rojiza. Cabeza grande y redondeada; disco frontal ligeramente punteado con estría frontal semicircular bien marcada. Pronoto más ancho que largo, arqueado y curvado a los lados con puntuación en la base; estrías laterales bien marcadas 


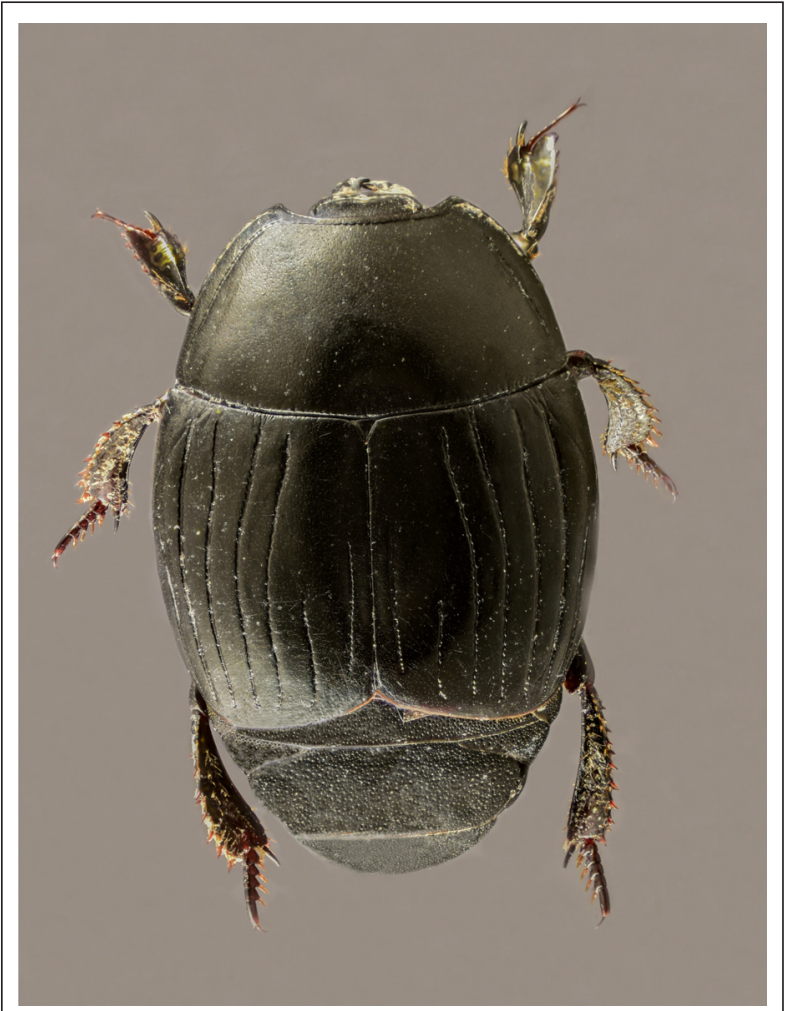

Fig. 3. Hister lissurus en vista dorsal. Foto por D. Hagopián.

y crenadas. Élitros curvos cortos, un poco más largos que el pronoto; estría subhumeral interna larga, sobrepasando la mitad del élitro; estrías 1 a 4 completas, bien marcadas, la 5 acortada a un tercio posterior del élitro, estría sutural más larga que la anterior. Propigidio liso con algunos puntos en los bordes. Pigidio completamente punteado. Protibias con tres dentículos, mesotibias y metatibias con 5-6 dentículos espinosos.

Distribución: Brasil, Paraguay, Argentina (Mazur, 2011) y Uruguay.

\section{Hister cavifrons Marseul, 1854 (Fig. 4)}

Cuerpo ovalado, poco convexo y finamente punteado (largo: 5,0 - 7,0 mm; ancho: 3,5 - $5 \mathrm{~mm}$ ). Color negro brillante, antenas marrones y maza gris. Cabeza pequeña y redondeada;frente con hoyuelo mediano y estría entera. Pronoto corto, más ancho que largo, arqueado y punteado en la base;estría lateral interna no interrumpida, curvada en la base; estría lateral externa recortada a la mitad. Élitros cortos, más largos que el pronoto. Bordes finamente punteados sin hoyuelos. Estría subhumeral interna desigualmente bifurcada. Estrías 1 a 4 completas, delgadas y punteadas, la 4 a veces interrumpida poco

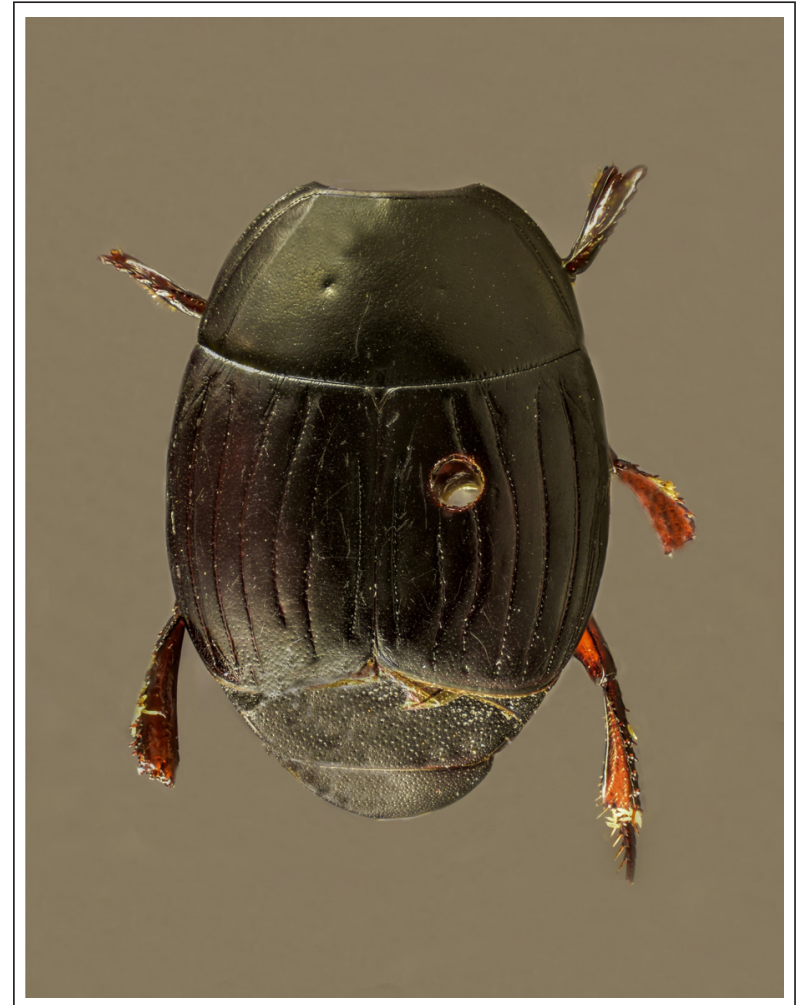

Fig. 4. Hister cavifrons en vista dorsal. Foto por D. Hagopián.

antes del ápice, la 5 casi llega a la mitad, la sutural, más larga que la anterior. Propigidio y pigidios cubiertos de grandes puntos dispersos. Puntuación más fina y compactada en los intervalos. Protibia con 5 dentículos pequeños y extremo bífido, mesotibias y metatibiascon 7 a 8 pares de dentículos espinosos.

Distribución: Venezuela (Marseul, 1853), Guatemala, Panamá (Blackwelder, 1945); América del Sur y Central (Mazur, 2011); Uruguay.

\section{Phelister pumilus Erichson, 1834 (Fig. 5)}

Cuerpo ovalado suborbicular, bastante convexo (largo: 2,5 mm; ancho: 1,5 mm). Color negro brillante, antenas y patas rojo claro, parte final de los élitros ferruginosos. Frente cóncava con puntuación, rodeada por una estría circular interrumpida al frente. Pronoto transverso, con borde anterior estrecho y arqueado y recurvado en la base, con un punto antescutelar; disco pronotal amplio con puntuación fina y densa; lateralmente con una franja de puntos más grandes y espaciados; estría marginal completa. Élitros anchos en la base, curvados a los lados y recto al final, un tercio más largos que el pronoto; estría subhumeral bien marcada, sobrepasando la mitad de los élitros; estrías 1 a 3 completas, la 4 acortada a la mitad, igual 


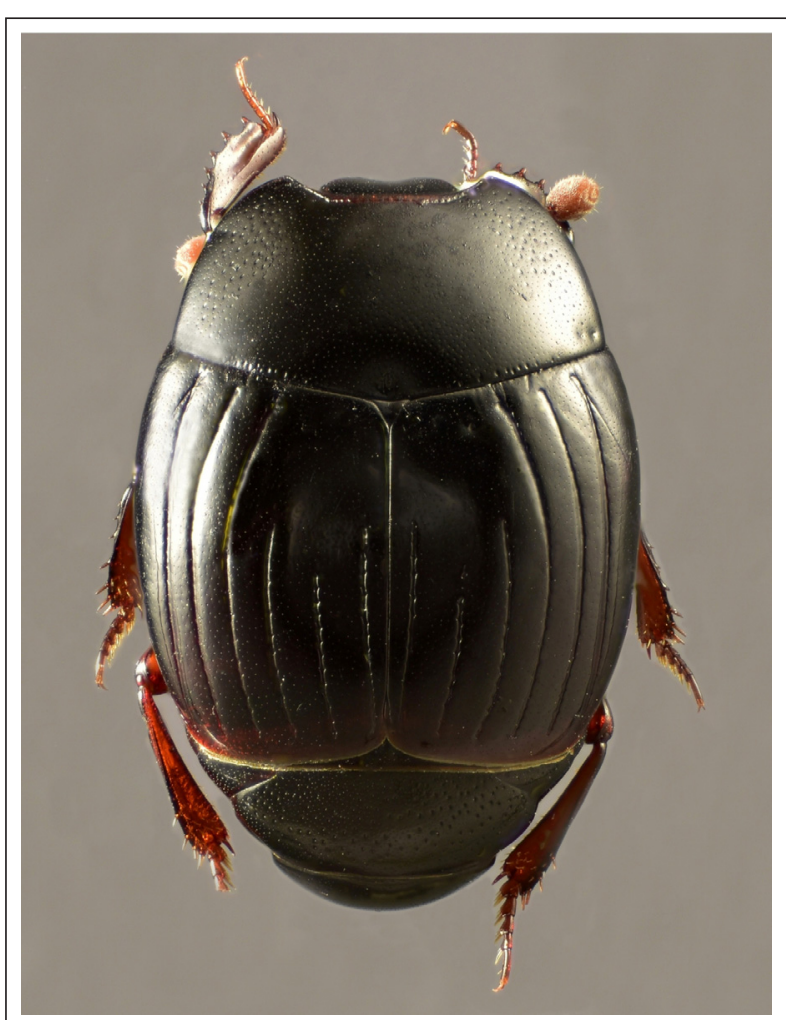

Fig. 5. Phelister pumilus en vista dorsal. Foto por D. Hagopián. a la sutural, 5 un poco más corta con dos grandes puntos en la base. Propigidio con puntuación vagamente dispersa. Pigidio con puntuación fina y densa. Protibias con 5 dentículos; mesotibias y metatibias con pocas espínulas.

Distribución: Brasil, Argentina, Venezuela (Mazur, 2011), Uruguay.

\section{Distribución potencial de la familia y de las especies más abundantes en Uruguay}

Fueron obtenidos 161 registros georreferenciados para la familia, de los cuales 80 , son puntos únicos (Fig. 6). Las especies más abundantes, E. azureus, $E$. connectens y $P$. pumilus presentaron respectivamente 17, 27 y 23 puntos únicos de registro (84\% del total).

Las variables seleccionadas para los modelos fueron $\mathrm{BIO} 3$ para la familia, BIO13 para $E$. azureus, $\mathrm{BIO} 11$ y $\mathrm{BIO} 3$ para $E$. connectens y $\mathrm{BIO} 1$ para $P$. pumilus (Tabla 1). La evaluación de los modelos indica que la discriminación es aceptable para los modelos de especies $(0.8>A \cup C>0.7)$, pero no así para el de la familia (0.7 > AUC) (Hosmer \& Lemeshow, 2000). Los valores de similaridad y CCR fueron siempre mayores a 0.6, mientras que los valores de sensibilidad fueron variados (Tabla 3).
Fig. 6. Distribución potencial de la familia Histeridae en Uruguay, obtenida por modelo basado en la función de favorabilidad. Los puntos representan los registros georreferenciados del taxón.

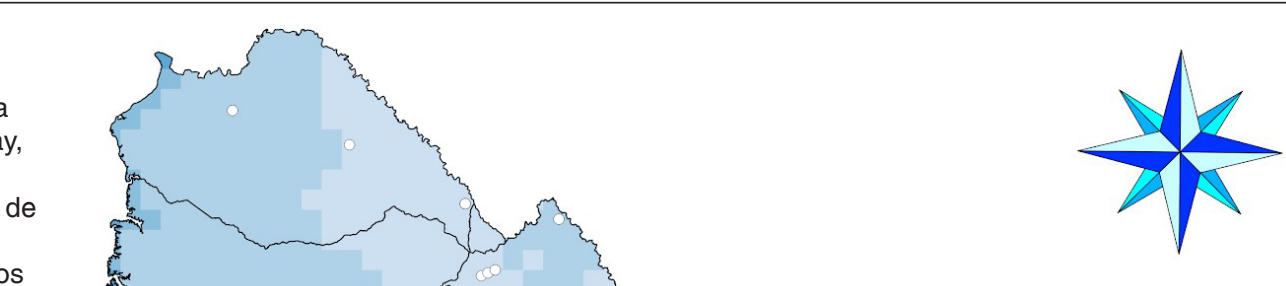

0 $150 \mathrm{~km}$

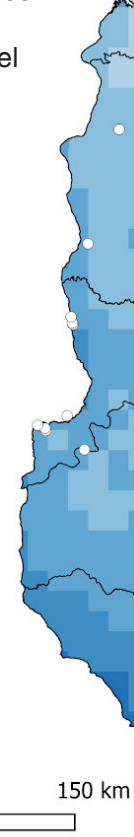
Favorabilidad 


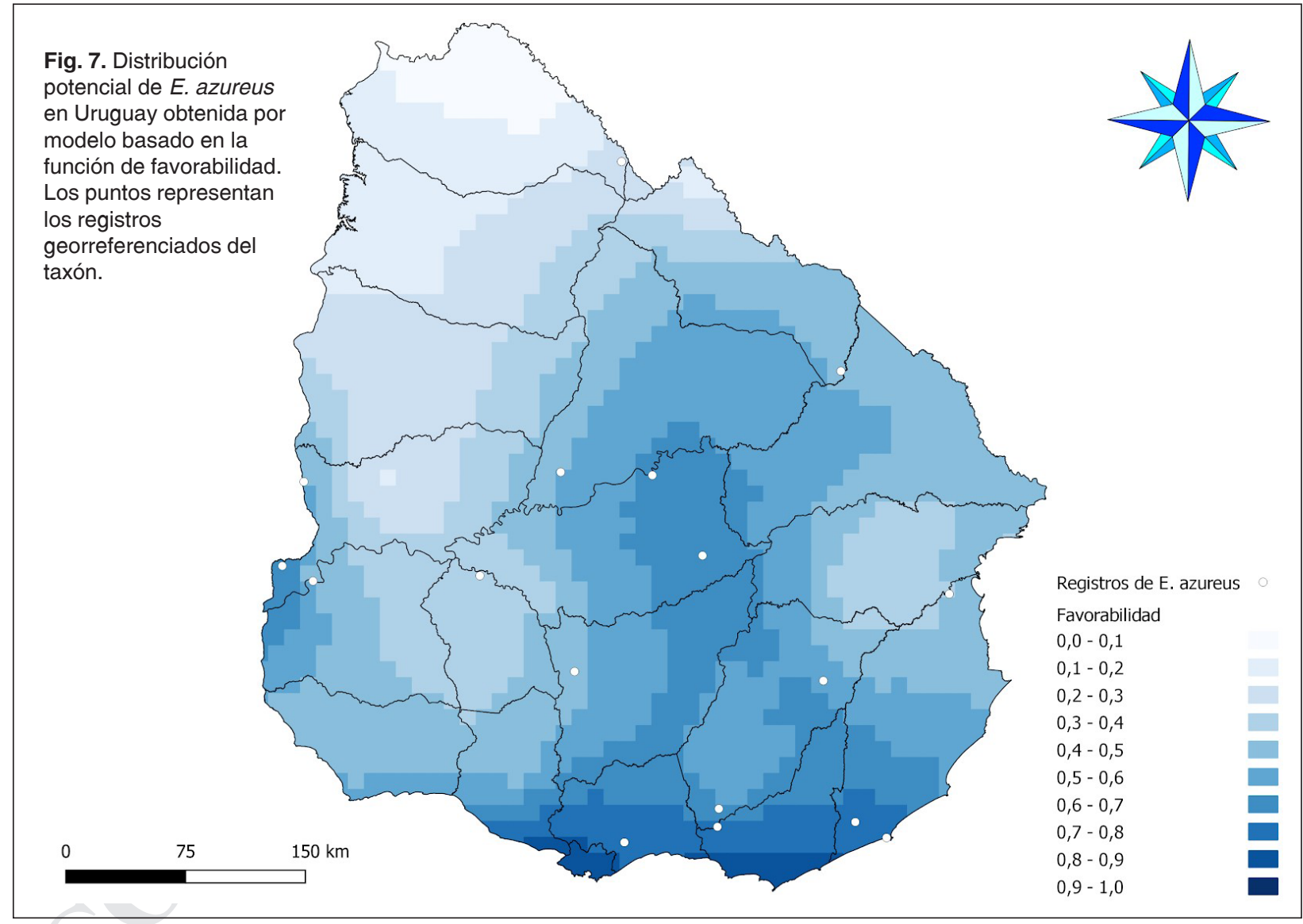

Fig. 8. Distribución potencial de $E$. connectens en Uruguay obtenida por modelo basado en la función de favorabilidad. Los puntos representan los registros georreferenciados del taxón.

0 75 $150 \mathrm{~km}$
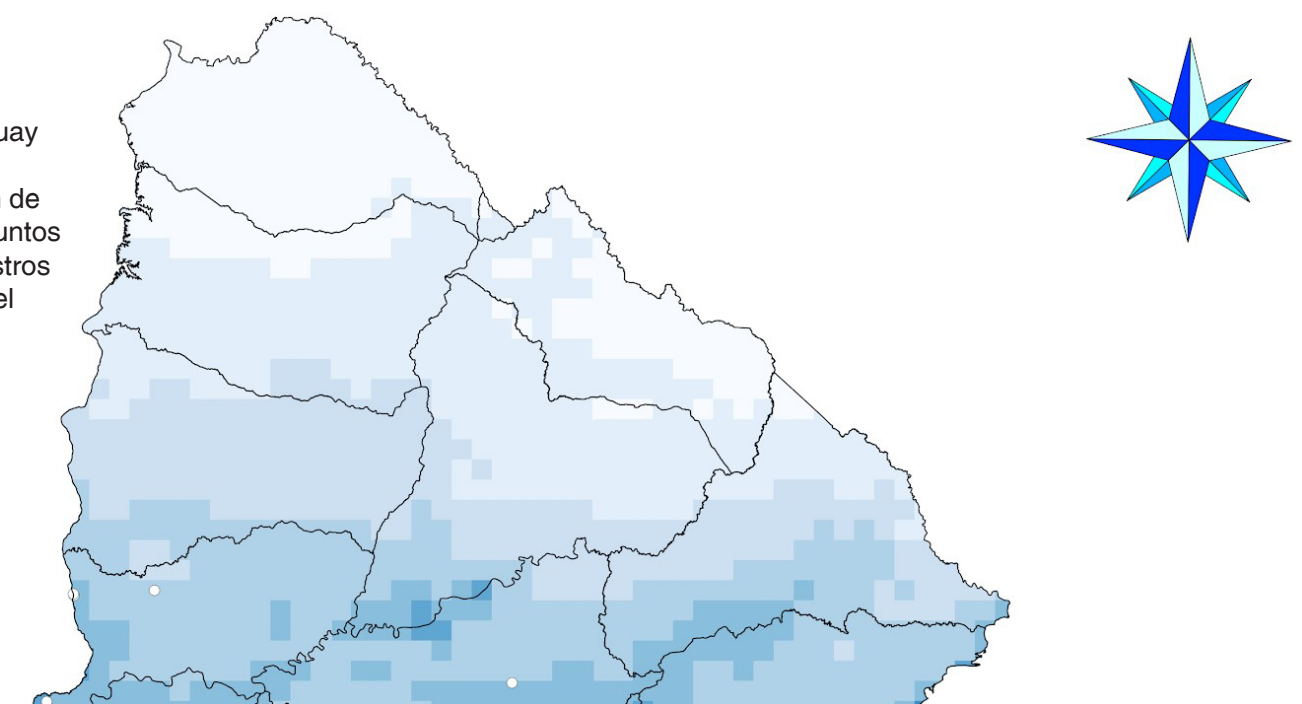

Registros de E. connectens Favorabilidad

$0,0-0,1$ $0,1-0,2$ $0,2-0,3$ $0,3-0,4$

$0,4-0,5$

$0,5-0,6$

$0,6-0,7$

$0,7-0,8$

$0,8-0,9$

$0,9-1,0$ 


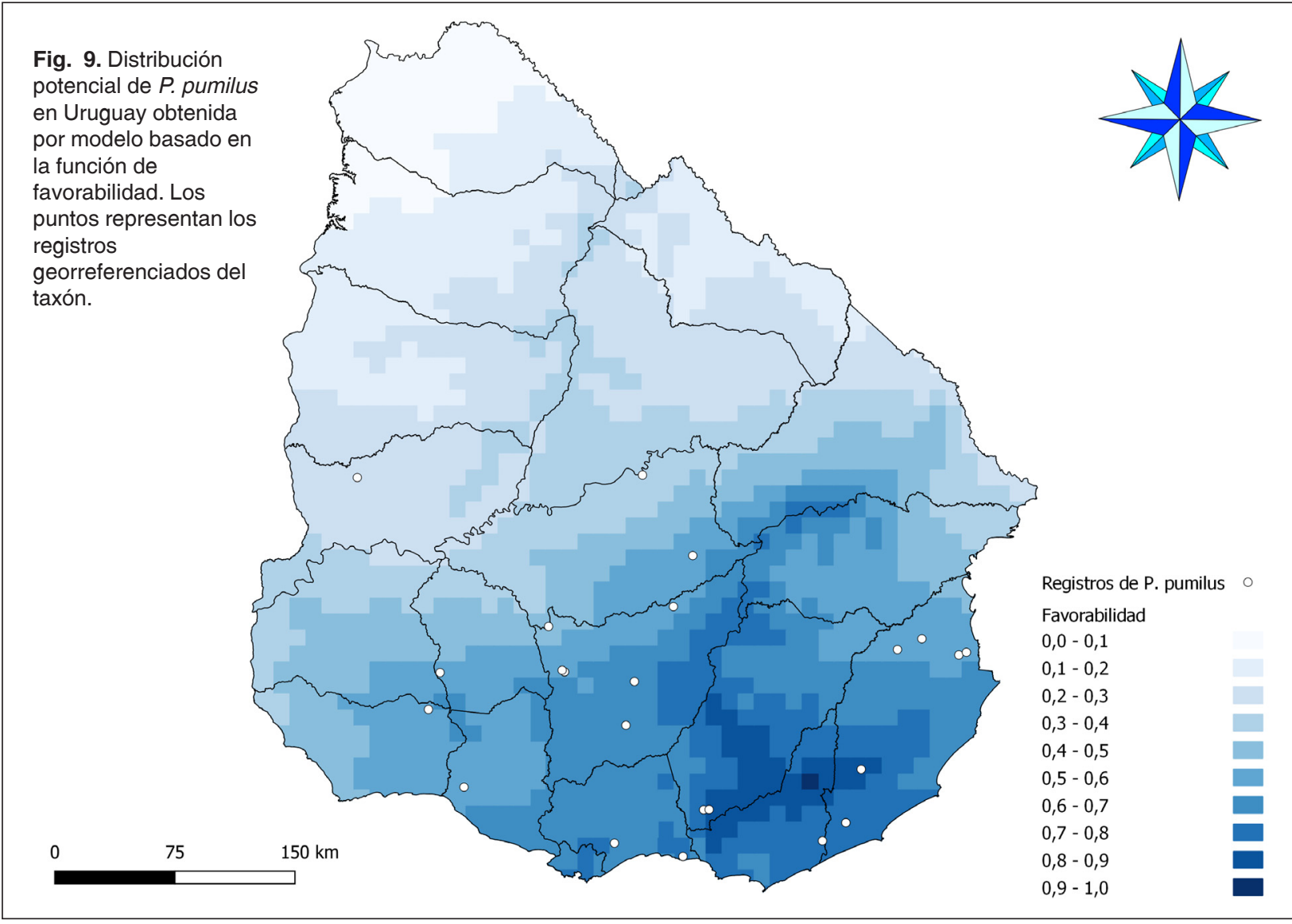

La familia presenta una amplia distribución en el territorio, que fue clasificado al menos como área de favorabilidad intermedia ( $F$ e» 0.2). Áreas de favorabilidad alta ( $F$ e» 0.8) fueron obtenidas en el litoral sur y este (Fig. 4). Áreas del norte de Uruguay fueron clasificadas como desfavorables para los modelos restantes, siendo áreas de alta favorabilidad el extremo sur para E. azureus (Fig. 7), región sur, porciones de la costa y Sierras del Este para $E$. connectens (Fig. 8), y Sierras del Este para P. pumilus (Fig. 9).

\section{DISCUSIÓN Y CONCLUSIÓN}

El presente trabajo agrega 10 especies de Histeridae al listado de Monné (1970), resultando en 37 el número actualizado de especies para Uruguay. De éstas, tres constituyeron nuevos registros para el país: Hister lissurus Marseul, 1854, Hister cavifrons Marseul, 1854 y Phelister pumilus Erichson 1834, siendo éste un aporte relevante del presente estudio. Veinte registros son exclusivamente de fuentes bibliográficas. Todas las especies de Histeridae que integran el listado son de distribución Neotropical.

El acervo del material de referencia de la FCE-Co se aumentó en 1139 ejemplares, 12 especies y 15 morfoespecies.

La mayoría de las especies (13) se encontraron exclusivamente en excremento, siete exclusivamente en cadáveres y 11 especies en ambos recursos. $E$. azureus, la especie más abundante, mostró una marcada preferencia por los cadáveres, lo cual reafirma su importancia para la Entomología Forense. Esta especie tiene una amplia distribución en el continente sudamericano (Mazur, 2011) y una amplia tolerancia térmica (Caneparo et al., 2015); en este estudio fue registrada en cinco ambientes diferentes, tanto abiertos como cerrados, nativos y exóticos, por lo cual se podría catalogar como una especie generalista en cuanto al hábitat. De la misma forma, E. connectens e Hister punctifer fueron generalistas en relación a su asociación con el hábitat.

El análisis de distribución espacial se realizó a nivel de familia dado que por un lado se encontraron varias especies en un mismo sitio y otras especies cuentan con un único registro. No se obtuvieron datos para los departamentos de Salto y Treinta y Tres. Para 
Tabla 3. Evaluación de los modelos de favorabilidad, con sus índices de discriminación y clasificación.

Índices de evaluación

Modelos de distribución

\begin{tabular}{|c|c|c|c|c|}
\hline \multirow{2}{*}{ Indices de evaluacion } & \multicolumn{4}{|c|}{ Miodelos de distribucion } \\
\hline & Histeridae & E. azureus & E. connectens & P. pumilus \\
\hline \multicolumn{5}{|l|}{ Discriminación } \\
\hline AUC & 0.6822057 & 0.7129317 & 0.7831232 & 0.7660068 \\
\hline \multicolumn{5}{|l|}{ Clasificación (umbral de 0.5) } \\
\hline Sensibilidad & 0.6440678 & 0.5882353 & 0.7391304 & 0.8636364 \\
\hline Especificidad & 0.6659353 & 0.6155496 & 0.6853147 & 0.6462366 \\
\hline CCR & 0.6652497 & 0.6153029 & 0.6859724 & 0.6487779 \\
\hline
\end{tabular}

Abreviaturas. AUC: área bajo la curva; CCR: tasa de clasificación correcta.

Cerro Largo existen solamente 2 registros bibliográficos sin localidades especificadas (Monné, 1970). Las áreas de mayor riqueza de especies fueron: Montevideo con 19 especies; Canelones, Florida y Río Negro con 11; Maldonado con 9; Rivera y Durazno con 8. Para todos los modelos, las variables bioclimáticas que explicaron la favorabilidad fueron un pequeño número del total de las variables utilizadas, y se obtuvo una marcada tendencia de que las áreas del Sur y Este sean clasificadas como altamente favorables mientras que las del Norte, desfavorables. Si bien las especies de Histeridae incluidas en los análisis son de amplia distribución Neotropical o regional en algunos casos, estos modelos están enfocados en la distribución local de estas especies. Estos resultados deben ser puestos a prueba en campo, con registro tanto de presencias como ausencias, en particular al Norte del Río Negro. Sugerimos que se incorporen en futuros modelos de distribución de la familia otras variables de carácter climático, topográfico, así como de cobertura y uso de suelo. Modelos de distribución, a nivel de la región o del continente, de grupos de especies de interés particular, como ser, el de agentes controladores de plagas, o de interés forense, podrían representar un aporte fundamental en la aplicación de su uso en el área respectiva. Por otra parte, modelos de distribución que se basen en el tipo de recurso utilizado por las especies, o en las preferencias de hábitat permitirían hacer inferencias de carácter ecológico o de relación con las actividades humanas. Los datos obtenidos en el presente estudio no permiten aún aplicar este tipo de modelos dado que para la mitad de las especies y la mayoría de las morfoespecies no se dispone de la información de la asociación con el hábitat. La presencia de mayor número de especies en pastizales y bosques de eucaliptus en este estudio se debe probablemente a que fueron los ambientes más relevados junto a los excrementos vacunos presentes en dichos ambientes.
La alta favorabilidad de las regiones Sur y Este de Uruguay para las especies de Histeridae pueden explicarse por el mayor esfuerzo de muestreo que históricamente se ha aplicado en estas zonas, mientras que el territorio nacional ha sido poco relevado al norte del Río Negro. En particular, no ha habido relevamientos previos enfocados específicamente en los coleópteros Histeridae y considerando que el presente trabajo tuvo un esfuerzo de muestreo acotado en espacio y tiempo, los resultados obtenidos no permiten aún hacer inferencias definitivas acerca de los patrones de distribución de riqueza de especies y de sus posibles causas. Este estudio tiene carácter preliminar; es necesario continuar con el relevamiento del grupo, aplicando además otros métodos de muestreo. El elevado número de morfoespecies aún por identificar reafirma la necesidad de reforzar los estudios de taxonomía de Histeridae en Uruguay.

\section{AGRADECIMIENTOS}

Se agradece a la Comisión Sectorial de Investigación Científica, Universidad de la República, por la financiación del proyecto "Coleópteros Histeridae de Uruguay: diversidad y distribución de las especies", a través del Programa de Apoyo a la Investigación Estudiantil. Agradecemos muy especialmente a Damián Hagopián por el aporte de ejemplares y por las fotografías de las nuevas especies registradas; al Dr. José Carlos Guerrero por la formación y asistencia brindada en los aspectos biogeográficos.

\section{BIBLIOGRAFÍA}

Aballay F.H., Arriagada G., Flores G.E. \& N.D. Centeno. 2013. An illustrated key to and diagnoses of the species of histeridae (coleoptera) associated 
with decaying carcasses in Argentina. ZooKeys, 261: 61-84. https://doi.org/10.3897/ zookeys.261.4226

Akaike H. 1998. Information theory and an extension of the maximum likelihood principle. En: Parzen, E., Tanabe, K., Kitagawa, G. (Eds.)Selected papers of Hirotugu Akaike, Springer Science, New York. 199-213 pp.

Almeida L.M. \& D.M. Mise. 2009. Diagnosis and key of the main families and species of South American Coleoptera of forensic importance. Revista Brasileira de Entomología, 53(2): 227244.

Arriagada G. 1987. Notas sinonímicas y datos distribucionales de Saprininae Neotropicales. Revista Chilena de Entomología, 15:61-70.

Arriagada G. 2018. Descripción de un nuevo género para SaprinusdolatusMarseul, 1862 (Coleoptera: Histeridae). Boletín del Museo Nacional de Historia Nacional, Chile, 67(2): 15-25.

Barbosa A.M., Brown J.A., Jiménez-Valverde A. \& R. Real. 2014. modEvA - ModelEvaluation and Analysis. Librería de R, versión 2.0.

Barbosa A.M. 2015. FuzzySim: applying fuzzy logic to binary similarity indices in ecology. Methods in Ecology and Evolution, 6: 853-858. Librería de $R$ versión 3.0.

Benjamini Y. \& Y. Hochberg. 1995. Controlling the false discovery rate: a practical and powerful approach to multiple testing. Journal of the Royal Statistical Society: Series B (Methodological), 57(1): 289-300.

Bentancourt C.M., Scatoni I.B. \& E. Morelli. 2009. Insectos del Uruguay. Ed. Hemisferio sur. Universidad de la República, Montevideo. 658 pp.

Blackwelder R.E. 1945. Checklist of the coleopterous insects of Mexico, Central America, the West Indies and South America. Bulletin of the United States National Museum, 185: 1-181.

Caneparo M.F., Fischer M.L. \& L.M. Almeida. 2015. Effect of temperatura on the life cycle of Euspilotus azureus (Coleoptera: Histeridae), a predator of forensic importance. Florida Entomologist, 100: 795-801.

Castro M., Centeno N. \& P. González-Vainer. 2019. An initial study of insect succession on pig carcasses in open pastures in the northwest of Uruguay. Forensic Science International, 302: 109837.

Caterino M.S. \& A.K. Tishechkin. 2019. A revision of the Phelister haemorrhous species group (Coleoptera, Histeridae, Exosternini). ZooKeys, 854: 41.

Cichino A. 1999. Rol de los coleópteros fimícolas como "enemigos naturales" de la mosca de los cuernos en la Argentina. Estado actual de su conocimiento y perspectivas futuras. Revista de la Sociedad Entomológica Argentina, 58 (1-
2): $172-179$.

Coletto-Silva A. \& D. da C.B. Freire. 2006. Hololepta (Leionota) reichii Marseul (Coleoptera, Histeridae), un nuevo enemigo natural para la meliponicultura en la Amazonía Central, Brasil. Revista Brasileira de Zoologia, 23(2): 588-591.

Degallier N., Mazur S., Tishechkin A.K. \& M.S. Caterino.2012. A revision of the genus Kaszabister Mazur (Histeridae, Histerinae, Exosternini). ZooKeys, 199: 71.

Erichson, W.F. 1834. Coleoptera und Lepidoptera, in Meyens, BeiträgezurZoologie, gesammelt auf einerReise um die erde, und W. Erichsons und $\mathrm{H}$. Burmeisters und Abbildungen der auf dieserReisegesammeltenInsecten. Nova Acta Academiae Caesareae Leopoldino-Carolinae, 219-248 pp.

Fernández L.A., Cabrera N.C. \& C.S. Gabellone. 2005. Los ejemplares tipo de Histeridae (Coleoptera), depositados en la colección del Museo de La Plata. Revista del Museo de la Plata. Universidad Nacional de la Plata, Facultad de Ciencias Naturales y Museo, La Plata, 47: 1-6.

Fick S. E. \& R. J. Hijmans. 2017. WorldClim 2: new 1$\mathrm{km}$ spatial resolution climate surfaces for global land areas. International Journal of Climatology, 37(12): 4302-4315.

Fielding A.H. \& J.F. Bell. 1997. A review of methods for the assessment of prediction errors in conservation presence/absence models. Environmental conservation, 24 (1): 38-49.

Gonçalves G. M. \& F. T. W. Leivas. 2017. Checklist de Histeridae do sul do Brasil (Insecta: Coleoptera: Staphyliniformia). Entomo Brasilis, 10 (3): 194213. https://doi.org/10.12741/ebrasilis.v10i3.700

Goñi B., Remedios M., González-Vainer P., Martínez M. \& C.R. Vilela. 2012. Species of Drosophila (Diptera: Drosophilidae) attracted to dung and carrion baited pitfall traps in the Uruguayan Eastern Serranías. Zoologia (Curitiba), 29(4): 308-317.

González-Vainer P. \& E. Morelli. 2008. Relevamiento de los coleópteros coprófilos y necrófilos de Sierra de Minas, Uruguay (Insecta: Coleoptera). Boletín de La Sociedad Zoológica Del Uruguay, 17: 20-33.

Greenberg B. \& M.L. Szyska. 1984. Immature stages and biology of fifteen species of Peruvian Calliphoridae (Diptera). Annals of the Entomological Society of America, 77(5): 488517.

Hastie T.J. \& D. Pregibon. 1992. Chapter 6. Statistical Models in S. Generalized Linear Models. Ed. by J.M. Chambers and T.J. Hastie, Wadsworth and Brooks/Cole.

Hosmer D.W. \& S. Lemeshow. 2000. Applied Logistic Regression, John Wiley \& Sons. New York.

Lackner T. 2010. Review of the Palaearctic genera of 
Saprininae (Coleoptera: Histeridae). Acta Entomologica Musei Nationalis Pragae, 50 (Suppl.): 1-254.

Lewis G. 1897. On new species of Histeridae, and notices of others. En: Taylor \& Francis (Eds) The Annals and magazine of natural history; zoology, botany, and geology, 356-364 pp. London, England.Ltd. https://www.biodiversitylibrary.org/ item/87729

Marseul S.A. de. 1853. Essai monographique sur la famille dês histérides. Annales de la Sociéte Entomologique de France, 3(1): 447- 553.

Marseul S.A. de. 1854. Essaimonographique sur la famille dê shistérides. Annales de la Sociéte Entomologique de France, 3(2): 161-311.

Marseul S.A. de. 1870. Description d'espéces nouvelles d'histérides. Annales de la Sociéte Entomologique de Belgique, 13: 54-138.

Mazur S. 2001. Review of the Histeridae (Coleoptera) of México. Dugesiana, 8(2): 17-66.

Mazur S. 2011. A concise catalogue of the Histeridae (Insecta: Coleoptera). Warzaw University of Life Sciences. SGGW Press, 322 pp.

Monné M.1970. Fauna de los coleópteros del Uruguay. Tesis Ing. Agr. Facultad de Agronomía, Montevideo, Uruguay.

Özdemir S. \& O. Sert. 2009. Determination of Coleoptera fauna on carcasses in Ankara province, Turkey. Forensic Science International, 183(1-3): 24-32. https://doi.org/10.1016/ j.forsciint.2008.09.018

Real R., Barbosa A.M., \& J.M. Vargas. 2006. Obtaining environmental favourability functions from logistic regression. Environmental and EcologicalStatistics, 13(2): 237-245.

Remedios M.L. 2014. Sucesión de entomofauna cadavérica en cuerpos de Sus scrofa L. (cerdo blanco) en un ambiente de bosque. Tesis de Maestría en Biología opción Zoología. Facultad de Ciencias, PEDECIBA. Montevideo.

Santoro P.H., P.M.O.J. Neves, T.M. Alexandre, S.A. Gavaguchi, \& L.F.A. Alves.2010. Carcinops troglodytes (Erichson) (Coleoptera: Histeridae) predando larvas de Alphitobius diaperinus (Panzer) (Coleoptera: Tenebrionidae) em aviários. Neotropical Entomology, 39(5): 831832.

Team R.C. 2013. R: A language and environment for statistical computing. Vienna, Austria.

The Document Foundation (2019). LibreOffice 6.3.0.4. Berlin, Germany. http:// www.libreoffice.org.

Fecha de Recepción: 14 de septiembre de 2020 Fecha de Aceptación: 27 de noviembre 2020 